\title{
UNIQUENESS OF SOLUTIONS OF PARTIAL DIFFERENTIAL EQUATIONS WHEN THE INITIAL SURFACE IS CHARACTERISTIC AT A POINT
}

\author{
LETITIA J. KORBLY ${ }^{1}$
}

\begin{abstract}
Uniqueness in the Cauchy problem for hyperbolic operators degenerate at a point on the initial surface depends on values of the coefficients of the lower order terms. If the operator $P$ is doubly characteristic at the origin with respect to the $t=0$ line, $P$ has uniqueness for functions which are smooth enough if the coefficient of the $D_{t}$ term does not lie in a certain discrete set of numbers.
\end{abstract}

Introduction. We consider operators of the form

$$
P=A(x, t) D_{t}^{2}+2 B(x, t) D_{x} D_{t}-C(x, t) D_{x}^{2}+\lambda(x, t) D_{t}+R_{1}
$$

where all the coefficients are real $C^{\infty}$ functions, $A$ is nonnegative, $C$ is positive, $R_{1}$ is a first order linear operator in $D_{x}$,

$$
D_{x}=\partial / \partial x \text { and } D_{t}=\partial / \partial t
$$

When $P$ is doubly characteristic at the origin with respect to the surface given by $t=0$, uniqueness of the solution to the problem:

$$
\begin{aligned}
P u & =F, \quad(x, t) \in \Omega, \text { a neighborhood of the origin, } t \geq 0, \\
u & =u_{0}(x), \quad \text { if } t=0, \\
D_{t} u & =u_{1}(x), \quad \text { if } t=0,
\end{aligned}
$$

is shown to depend on the values of the lower order terms, principally $\lambda$. If $\lambda$ lies outside a certain discrete set of values the solutions to the problem will be unique whenever $\mu$ is smooth enough.

If $\lambda$ lies in this discrete set of values, uniqueness will depend on $R_{1}$. The dependence on the lower order terms is very complex. There is always uniqueness when

$$
\lambda_{0}=\lambda-D_{x} B-D_{t} A / 2 \geq 0 .
$$

As $\lambda_{0}$ becomes more negative, $u$ must be assumed to be smoother to get the same result.

There are some values of the coefficients for which no uniqueness results can be obtained. If $c>0$,

$$
P u=x^{2} D_{t}^{2} u-c^{2} D_{x}^{2} u-(2 n+1) c D_{t} u
$$

Received by the editors December 10, 1981.

1980 Mathematics Subject Classification. Primary 35L80, 35A07, 35L70; Secondary 35L15.

Key words and phrases. Hyperbolic PDE, Cauchy problem, doubly characteristic .

${ }^{1}$ Part of this work is based on the author's thesis written under F. Treves at Rutgers University 
and

$$
\tilde{X} u=x D_{t} u-c D_{x} u
$$

then

$$
P \tilde{X}^{N} h\left(c t-x^{2} / 2\right)=0, \quad h \in C_{c}^{\infty}(R) .
$$

The assertion is proved by taking the support of $h$ to be the positive halfline. Finally, if $P$ is as in (3), $P+\gamma$ is shown to have uniqueness if $\gamma$ is a nonzero function.

The operators. $P$ is characteristic with respect to the initial surface at the origin when $A$ vanishes at the origin, and doubly characteristic with respect to this surface when both $B$ and grad $A$ also vanish there.

In order to use the concatenation technique of Visik and Grusin, [12], it is necessary to factor the principal part of the operator in a neighborhood of the origin which we will take to be $\Omega$. The following hypotheses are assumed:

(Ha) There is a $C^{\infty}$ function, $a$, such that

$$
A=a^{2} \text { and } D_{x} a=a_{x}>0 \text {. }
$$

(Hb) There is a $C^{\infty}$ function, $b$, such that

$$
B=b a \text {. }
$$

Then

$$
P=a^{2} D_{t}^{2}+2 a b D_{t} D_{x}+b^{2} D_{x}^{2}-\left(C+b^{2}\right) D_{x}^{2}+\lambda D_{t}+R_{1}
$$

Let

$$
c^{2}=C+b^{2}, \text { where } c>0
$$

Define

$$
\tilde{Y}=a D_{t}+b D_{x}+c D_{x} \text { and } \tilde{X}=a D_{t}+b D_{x}-c D_{x}
$$

So

$$
P=\tilde{Y} \tilde{X}+\lambda^{\prime} D_{t}+R_{2},
$$

where $R_{2}$ is a first order operator in $D_{x}$ and

$$
\lambda^{\prime}=\lambda-(b+c) a_{x} .
$$

This form is used in the concatenations. The commutator, $[\tilde{Y}, \tilde{X}]$, of $\tilde{Y}$ and $\tilde{X}$ acting on a function is

$$
[\tilde{Y}, \tilde{X}] u=(\tilde{Y} \tilde{X}-\tilde{X} \tilde{Y}) u=\left(2 a_{x} c D_{t}+R_{3}\right) u,
$$

where $R_{3}$ is a first order operator in $D_{x}$. The hypotheses (1) and (Ha) guarantee $a_{x} c>0$.

DEFINITION 1. The following norms are used:

$$
\|u\|^{2}=\|u\|_{0}^{2}=\iint_{\Omega}|u|^{2} d x d t, \quad u \in \mathcal{H}^{2}(\Omega) .
$$

Then

$$
\|u\|_{N+1}^{2}=\left\|D_{t} u\right\|_{N}^{2}+\sigma^{2}\|u\|_{N}^{2}+\left\|D_{x} u\right\|_{N}^{2} .
$$

The following norm is taken from [1]:

$$
\|u\|_{1,1}^{2}=\left\|a D_{t} u\right\|_{0}^{2}+\sigma^{2}\|a u\|_{0}^{2}+\left\|D_{x} u\right\|_{0}^{2} .
$$


Note that for all $u$ in $C_{c}^{\infty}(\Omega)$, and $\sigma>\sigma_{0}$ :

$$
\|u\|_{1,1} \leq\|u\|_{1} \text {. }
$$

Also, if $u \in C_{c}^{\infty}(\Omega)$,

$$
\left\|\left(D_{t}+\sigma\right) u\right\|^{2}=\left\|D_{t} u\right\|^{2}+\sigma^{2}\|u\|^{2} .
$$

Similar statements are true when other norms are used.

DEFINITION 2. An operator, $P$, has $(U C P)_{N}$ in an open set, $\Omega$, if, whenever $u$ is an element of $H^{N}(\Omega)$, and supp $u \cap\{t \leq 0\}$ is compact, then $P u=0$ in $\Omega$, implies $u=0$ in a neighborhod of supp $u \cap\{t \leq 0\}$.

Let $\Omega^{-}=\Omega \cap\{t \leq 0\}$. The following lemma is only stated because its proof is an easy modification of that in [8].

LEMMA 1. If there are constants $c$ and $\sigma_{0}$ such that for every function $v$ in $C_{c}^{\infty}(\Omega)$, and every $\sigma>\sigma_{0}$, the Carleman estimate

$$
C\left\|e^{-\sigma t} P v\right\|_{N} \geq\left\|e^{-\sigma t} v\right\|_{0}
$$

holds, then $P$ has $(U C P)_{N+m}$. Here $m$ is the order of $P$.

Define

$$
P_{\phi} u=\exp (-\sigma t) P(\exp (\sigma t) u)
$$

The substitution

$$
v=\exp (\sigma t) u
$$

shows that (6) is equivalent to the following: there is a constant $c$, such that

$$
c\left\|P_{\phi} u\right\|_{N} \geq\|u\|_{0}, \quad u \in C_{c}^{\infty}(\Omega), \sigma>\sigma_{0} .
$$

This is the estimate used in proving the theorem.

Proof of the theorem when $\lambda_{0}$ is positive. Let $A_{t}$ be the partial of $A$ with respect to $t, B_{x}$, the partial of $B$ with respect to $x$, and $C_{t}$ and $C_{x}$ be the partials of $C$ with respect to $t$ and $x$ respectively.

LEMMA 2. If $P$ is as in (1) where $A$ is nonnegative and $C$ is positive, and

$$
0<L<\lambda_{0}=\lambda(0,0)-B_{x}(0,0)-A_{t}(0,0) / 2,
$$

then there are $c>0$ and $\sigma_{0}$, depending only on $L$ and the coefficients of $P$ such that if $\Omega$ is a sufficiently small neighborhood of the origin,

$$
\left\langle P_{\phi} u,\left(D_{t}+\sigma\right) u\right\rangle \geq c \sigma\|u\|_{1,1}^{2}+L\|u\|_{1}^{2}, \quad u \in C_{c}^{\infty}(\Omega), \sigma>\sigma_{0} .
$$

PROOF. Integration by parts in $D_{t}$ shows

(7) $\left\langle A\left(D_{t}+\sigma\right)^{2} u,\left(D_{t}+\sigma\right) u\right\rangle=\sigma\left\|A^{1 / 2}\left(D_{t}+\sigma\right) u\right\|^{2}-\left\langle A_{t} / 2\left(D_{t}+\sigma\right) u,\left(D_{t}+\sigma\right) u\right\rangle$

whenever $u$ is in $C_{c}^{\infty}(\Omega)$.

Integration by parts in $D_{x}$ shows that if $u \in C_{c}^{\infty}(\Omega)$,

$$
\left\langle 2 B D_{x}\left(D_{t}+\sigma\right) u,\left(D_{t}+\sigma\right) u\right\rangle=-\left\langle B_{x}\left(D_{t}+\sigma\right) u,\left(D_{t}+\sigma\right) u\right\rangle .
$$

An integration by parts in $D_{t}$ followed by another in $D_{x}$ shows if $u \in C_{c}^{\infty}(\Omega)$,

$$
\begin{aligned}
-\left\langle C D_{x}^{2} u,\right. & \left.\left(D_{t}+\sigma\right) u\right\rangle \\
& =\sigma\left\|C^{1 / 2} D_{x} u\right\|^{2}-\left\langle\left(C_{t} / 2\right) D_{x} u, D_{x} u\right\rangle+\left\langle\left(C_{x} / 2\right) D_{x} u,\left(D_{t}+\sigma\right) u\right\rangle .
\end{aligned}
$$


We use (1), (7), (8), and the last equation, (9), to get if $u \in C_{c}^{\infty}(\Omega)$,

$$
\begin{aligned}
\left\langle P_{\phi} u,\left(D_{t}+\sigma\right) u\right\rangle=\sigma & \left\|a\left(D_{t}+\sigma\right) u\right\|^{2}+\left\|\left(\sigma C-C_{x} / 2\right)^{1 / 2} D_{x} u\right\|^{2} \\
& +\left\|\left(\lambda_{0}\right)^{1 / 2}\left(D_{t}+\sigma\right) u\right\|^{2}+\left\langle R_{4} u,\left(D_{t}+\sigma\right) u\right\rangle
\end{aligned}
$$

where $R_{4}$ is a first order operator in $D_{x}$.

Schwarz's inequality implies that for some $R$ and any positive $\epsilon$,

$$
\left\langle R_{4} u,\left(D_{t}+\sigma\right) u\right\rangle \leq \epsilon\left\|\left(D_{t}+\sigma\right) u\right\|^{2}+1 /(2 \epsilon)\|R u\|^{2}, \quad u \in C_{c}^{\infty}(\Omega) .
$$

Fix $\epsilon$ so that $L=\lambda_{0}-\epsilon>0$ after shrinking $\Omega$ if necessary. Then choose $\sigma_{0}$ so that $\left.\sigma_{0} C-C_{x} / 2-R / 2 \epsilon\right)$ is positive. This implies that the first three terms on the right in (10) dominate the last term on the right, which proves the lemma.

COROLLARY. If $P$ is as in (1) and $(\mathrm{Ha})$ and $(\mathrm{Hb})$ hold, the conclusion of Lemma 2 is true if

$$
\lambda-a_{x} b>L>0 .
$$

ProOF. Both $a b_{x}$ and $A_{t} / 2=a a_{t}$ vanish at the origin.

Proof of the theorem when $\lambda_{0}$ is negative. It is easy to show that $P_{\phi} u$ dominates $Y^{N} u$ if $\lambda_{0}$ is not less than $-2 N a_{x} c$, where

$$
Y u=e^{-\sigma t} \tilde{Y}\left(e^{\sigma t} u\right), \quad u \in C_{c}^{\infty}(\Omega)
$$

and

$$
Y^{N+1} u=Y Y^{N} u
$$

But no inequality which is uniform in $\sigma$ bounds $Y u$ away from 0 in a neighborhood of the origin. To prove the theorem when $\lambda_{0}<0$, more structure is necessary. The next lemma handles the commutators between $Y^{N}$ and $P_{\phi}$. Let

$$
X u=e^{-\sigma t} X\left(e^{\sigma t} u\right), \quad u \in C_{c}^{\infty}(\Omega) .
$$

LEMMA 3. If $P$ is as in (1) and $(\mathrm{Ha})$ and $(\mathrm{Hb})$ are satisfied, then

$$
Y^{N} P_{\phi} u=\left(P_{\phi}+N[Y, X]\right) Y^{N} u+\sum_{k<N} L_{k} Y^{k} u, \quad u \in C_{c}^{\infty}(\Omega) .
$$

Here $L_{k}$ is a first order operator in $D_{x}$ and $D_{t}$.

Proof. The proof is by induction. The lemma is clearly true when $N=0$. The induction hypothesis is (11). Then if $u \in C_{c}^{\infty}(\Omega)$,

$$
Y^{N+1} P_{\phi} u=Y\left[\left(X Y+\lambda^{\prime}\left(D_{t}+\sigma\right)+R_{2}+N[Y, X]\right) Y^{N} u+\sum_{k<N} L_{k} Y^{k} u\right] .
$$

$Y$ is commuted with the rest of the right-hand side above. The commutator satisfies

$$
[Y, X Y]=[Y, X] Y \text {. }
$$

All the other commutators are first order. This recovers the inductive hypothesis. The lemma follows.

COROLlarY. If $P$ is as in (1) and satisfies $(\mathrm{Ha})$ and $(\mathrm{Hb})$, and if

$$
\lambda(0,0)-a_{x}(0,0) b(0,0)=\lambda_{0}(0,0)>-2 N a_{x}(0,0) c(0,0)
$$

and

$$
P_{\phi}^{N}=P_{\phi}+N[Y, X]
$$

then there is some neighborhood $\Omega$ of the origin where $P^{N}$ satisfies the hypotheses of Lemma 2. 
Proof. Define

$$
\lambda_{N}=\lambda_{0}+2 N a_{x} c
$$

then the hypothesis of the corollary implies that in some neighborhood of the origin $\lambda_{N}$ is strictly positive, and must be bounded away from 0 in some neighborhood of the origin which is taken to be $\Omega$. But

$$
P_{\phi}^{N}=X Y+\lambda_{N} D_{t}+R_{N}
$$

where $R_{N}$ is a first order operator in $D_{x}$. This proves the corollary.

Now the main theorem will be proved.

THEOREM 1. If $P$ is as in (1) and $(\mathrm{Ha})$ and $(\mathrm{Hb})$ are satisfied then $P$ has $(U C P)_{N+2}$ if

$$
\lambda^{\prime}+2 k a_{x}(0,0) c(0,0) \neq 0 \text { for any } k=1,2,3, \ldots
$$

and

$$
\lambda_{0}+2 N a_{x}(0,0) c(0,0)>0 .
$$

Proof. Lemma 3 implies that there are constants $c, r$, and $\sigma_{0}$ such that if $u \in C_{c}^{\infty}(\Omega)$ and $\sigma>\sigma_{0}$,

$$
c\left\|P_{\phi} u\right\|_{N} \geq\left\|Y^{N} P_{\phi} u\right\|_{0} \geq\left\|P_{\phi}^{N} Y^{N} u\right\|_{0}-S_{0}
$$

Here

$$
S_{J}=\sigma^{J} \sum_{k<N-J} r_{k}\left\|Y^{k} u\right\|_{1}
$$

The corollary to Lemma 3 and (h2) establish that $P^{N}$ satisfies the hypothesis of Lemma 2, so there are constants $c$ and $\sigma_{0}$, such that if $u \in C_{c}^{\infty}(\Omega)$ and $\sigma>\sigma_{0}$,

$$
c\left\langle P_{\phi}^{N} Y^{N} u,\left(D_{t}+\sigma\right) Y^{N} u\right\rangle \geq \sigma\left\|Y^{N} u\right\|_{1,1}^{2}+\left\|Y^{N} u\right\|_{1}^{2}
$$

Since

$$
\left\|\left(D_{t}+\sigma\right) v\right\|_{0} \leq\|v\|_{1}
$$

and because the 1,1 norm is smaller than the 1 norm, (14) implies there are constants, $c$ and $\sigma_{0}$, such that if $u \in C_{c}^{\infty}(\Omega), \sigma>\sigma_{0}$ and $N \geq 1$,

$$
c\left\|P_{\phi} u\right\|_{N} \geq \sigma\left\|Y^{N} u\right\|_{1,1}+\left\|Y^{N} u\right\|_{1} \text {. }
$$

Substitution of the last inequality into (13) implies there is a constant $c$ such that if $u \in C_{c}^{\infty}(\Omega)$ and $\sigma>\sigma_{0}$,

$$
c\left\|P_{\phi} u\right\|_{N} \geq \sigma\left\|Y^{N}\right\|_{1,1}+\left\|Y^{N} u\right\|_{1}-S_{0} .
$$

This inequality is the first step in an inductive argument. The inductive hypothesis is that there are constants $c, r$, and $\sigma_{0}$, depending on $J, N$, and $P$, but not on $u \in C_{c}^{\infty}(\Omega)$ or $\sigma>\sigma_{0}$, such that

$$
c\left\|P_{\phi} u\right\|_{N} \geq \sigma^{J}\left(\sigma\left\|Y^{N-J} u\right\|_{1,1}+\left\|Y^{N-J} u\right\|_{1}\right)-S_{J} .
$$

We now try to recover the inductive hypothesis for $J+1$. The inductive hypothesis for $J$ implies there are constants $c^{\prime}, r^{\prime}$, and $\sigma_{0}$ such that if $u \in C_{c}^{\infty}(\Omega)$ and $\sigma>\sigma_{0}$,

$$
c^{\prime}\left\|P_{\phi} u\right\|_{N} \geq \sigma^{J+1}\left\|X Y Y^{N-J-1} u\right\|_{0}-S_{J} .
$$


But the definition of the norms implies there is a constant $c^{\prime \prime}$ such that

$$
c^{\prime \prime}\left\|P_{\phi} u\right\|_{N} \geq \sigma^{J+1}\left\|Y^{N-J-1} P_{\phi} u\right\|_{0}, \quad u \in C_{c}^{\infty}(\Omega), \sigma>\sigma_{0} .
$$

Lemma 3 applied to the right-hand side of the inequality above implies the existence of constants $c^{\prime \prime \prime}, r^{\prime \prime}$, and $\sigma_{0}$ such that if $u \in C_{c}^{\infty}(\Omega)$ and $\sigma>\sigma_{0}$,

$$
c^{\prime \prime \prime}\left\|P_{\phi} u\right\|_{N} \geq \sigma^{J+1}\left\|P_{\phi}^{N-J-1} Y^{N-J-1} u\right\|_{0}-S_{J+1} \text {. }
$$

Subtracting (16) from the last inequality gives the following for $u \in C_{c}^{\infty}(\Omega)$ and $\sigma>\sigma_{0}:$ if $\underline{c}=c^{\prime}+c^{\prime \prime \prime}$, and $\lambda_{N-J-1}$ is as in (12),

$$
c\left\|P_{\phi} u\right\|_{N} \geq \sigma^{J+1}\left\|\lambda_{N-J-1}\left(D_{t}+\sigma\right) Y^{N-J-1} u\right\|_{0}-\sigma^{J} r^{\prime}\left\|Y^{N-J-1} u\right\|_{1}-S_{J+1} \text {. }
$$

Here $r^{\prime \prime}$ and $\sigma_{0}$ have been increased to absorb all the terms of $S_{J}$ but the $(N-J-1)$ st. The $(N-J-1)$ st term is the one which must be dominated to recover the inductive hypothesis.

Since (h2) guarantees that $\lambda_{N-J-1}=0$ in $\Omega$, we can add a multiple, $m$, of the last inequality to (17) to get for each $u \in C_{c}^{\infty}(\Omega)$ and $\sigma>\sigma_{0}$,

$$
\underline{c}^{\prime}\left\|P_{\phi} u\right\|_{N} \geq \sigma^{J+1}\left\|P_{\phi}^{g} Y^{N-J-1} u\right\|_{0}-\sigma^{J} m r^{\prime}\left\|Y^{N-J-1} u\right\|_{1}-S_{N+1},
$$

where $\underline{c}^{\prime}=m \underline{c}+c^{\prime \prime \prime}$. The multiple $m$ is chosen so that $P_{\phi}^{g}$ satisfies the hypothesis of Lemma 2. This now implies that for some new $\underline{c}^{\prime \prime}$ and $\sigma_{0}$, if $u \in C_{c}^{\infty}(\Omega)$ and $\sigma>\sigma_{0}$,

$$
\begin{gathered}
\underline{c}^{\prime \prime}\left\|P_{\phi} u\right\|_{N} \geq \sigma^{J+1}\left(\sigma\left\|Y^{N-J-1} u\right\|_{1,1}+\left\|Y^{N-J-1} u\right\|_{1}\right) \\
-\sigma^{J} r\left\|Y^{N-J-1} u\right\|_{1}-S_{N+1} .
\end{gathered}
$$

If $\sigma_{0}$ is taken bigger than $r$, then the inductive hypothesis is recovered. This proves the estimate. The theorem follows from Lemma 1.

With the introduction of more machinery, as in [ 1 and 2], a slightly stronger theorem can be proved. Is is possible to show that the conditions of Theorem 1 imply $P$ has $(U C P)_{N+1}$. It is probably not possible to conclude that $P$ has uniqueness for functions with continuous second partials when $\lambda_{0}$ is negative. It is also possible to strengthen the conclusion of Theorem 2 slightly, but the gain is small compared to the increase in difficulty of the proof.

COROLlARY. If $P$ is as in (1) and (Ha) and (Hb) are satisfied, then $P$ has $(U C P)_{N+2}$ if

$$
\lambda \neq(b-(2 k-1) c) a_{x}, \quad k=1,2,3, \ldots
$$

and

$$
\lambda>(b-2 N c) a_{x}
$$

PROOF. The proof that (h2)' is equivalent to (h2) is exactly as in the corollary to Lemma 2. The equivalence of (h1)' to (h1) follows directly from (2), the definition of $\lambda_{0}$.

COROLlARY 2. If $P$ is as in (1) and $(\mathrm{Ha})$ and $(\mathrm{Hb})$ hold, then $P$ has $(U C P)_{N+2}$ if for every positive integer $k$, when $t=0$,

$$
\lambda(0,0) \neq \lim _{x \rightarrow 0}\left\{A_{x}\left[B-(2 k-1)\left(A C+B^{2}\right)^{1 / 2}\right] /(2 A)\right\}
$$

and when $t=0$,

$$
\lambda(0,0)>\lim _{x \rightarrow 0}\left\{A_{x}\left[B-2 N\left(A C+B^{2}\right)^{1 / 2}\right] /(2 A)\right\} .
$$


ProOF. This follows directly from the definitions of $a, b$, and $c$. We have

$$
a_{x}=A_{x} /\left(2 A^{1 / 2}\right) \text { and } b a_{x}=A_{x} B /(2 A) .
$$

Also

$$
c a_{x}=A_{x}\left(A C+B^{2}\right)^{1 / 2} /(2 A) .
$$

The final theorem shows the complex dependence of uniqueness on the terms $R_{N}$ when $\lambda$ violates $(\mathrm{h} 1)$.

THEOREM 2. Let $\gamma$ be a nonvanishing function,

$$
\begin{gathered}
P u=(X Y+N[Y, X]+\gamma) u, \\
Y \gamma=0 \text { and }[Y,[Y, X]]=0 .
\end{gathered}
$$

Then $P$ has $(U C P)_{N+4}$.

ProOF. Application of Lemma 2 shows there are constants $c$ and $\sigma_{0}$, depending on $P$ but not on $u \in C_{c}^{\infty}(\Omega)$ or $\sigma>\sigma_{0}$, such that

$$
c\left\|P_{\phi} u\right\|_{N+2} \geq\left\|Y^{N+1} P_{\phi} u\right\|_{1}=\left\|P_{\phi}^{N+1} Y^{N+1} u\right\|_{1} .
$$

Here $P^{N+1}$ satisfies the hypothesis of Lemma 2. If for $u \in C_{c}^{\infty}(\Omega)$,

$$
\|u\|_{2,1}^{2}=\left\|u_{t}\right\|_{1,1}^{2}+\left\|u_{x}\right\|_{1,1}^{2}+\sigma^{2}\|u\|_{1,1}^{2},
$$

an easy extension of Lemma 2 shows there are constants $c$ and $\sigma_{0}$ such that

$$
c\left\|P_{\phi} u\right\|_{N+2} \geq \sigma\left\|Y^{N+1} u\right\|_{2,1}+\left\|Y^{N+1} u\right\|_{2}, \quad u \in C_{c}^{\infty}(\Omega), \sigma>\sigma_{0} .
$$

This implies there are $c^{\prime}$ and $\sigma_{0}$ such that if $u \in C_{c}^{\infty}(\Omega)$ and $\sigma>\sigma_{0}$,

$$
c^{\prime}\left\|P_{\phi} u\right\|_{N+2} \geq \sigma\left\|X Y Y^{N} u\right\|_{1} .
$$

But the definitions of the norms imply that for some $c$ and $\sigma_{0}$, if $u \in C_{c}^{\infty}(\Omega)$ and $\sigma>\sigma_{0}$

$$
c^{\prime \prime}\left\|P_{\phi} u\right\|_{N+2} \geq \sigma\left\|Y^{N} P_{\phi} u\right\|_{1}=\sigma\left\|P_{\phi}^{N} Y^{N} u\right\|_{1} .
$$

Here a whole derivative has been lost. Subtracting (11) from (18) implies that if $u \in C_{c}^{\infty}(\Omega)$ and $\sigma>\sigma_{0}$,

$$
\left(c^{\prime \prime}+c^{\prime}\right)\left\|P_{\phi} u\right\|_{N+2} \geq \sigma|\gamma(0,0) / 2|\left\|Y^{N} u\right\|_{1} .
$$

The induction starts here. The inductive hypothesis is: there are $c$ and $\sigma_{0}$ such that if $u \in C_{c}^{\infty}(\Omega)$ and $\sigma>\sigma_{0}$,

$$
c\left\|P_{\phi} u\right\|_{N+2} \geq \sigma^{J+1}\left\|Y^{N-J} u\right\|_{1} .
$$

The induction is similar to that in Theorem 1. The hypotheses of this theorem guarantee all the commutators except $[Y, X]$ vanish. When $J=N$, an estimate which proves the theorem is obtained. 


\section{REFERENCES}

1. L. Boutet de Monvel and F. Treves, On a class of pseudodifferential operators with double characteristics, Invent. Math. 24 (1974), 1-34.

2. $\ldots$, On a class of systems of pseudodifferential operators with double characteristics, Comm. Pure Appl. Math. 27 (1974), 59-89.

3. M. S. Baouendi and C. Goulaouic, Cauchy problems with characteristic initial surface, Comm. Pure Appl. Math. 26 (1973), 555-575.

4. A. Gilioli and F. Treves, An example in the solvability theory of linear PDE's, Amer. J. Math. 96 (1974), 367-385.

5. L. Hormander, Linear partial differential operators, 3rd printing, Springer-Verlag, Berlin and New York, 1969.

6. A. Menikoff, Uniqueness of the Cauchy problem for a class of partial differential operators with double characteristics, Indiana Univ. Math. J. 25 (1976), 1-21.

7. F. Treves, Concatenations of second order evolution equations applied to local solvability and hypoellipticity, Comm. Pure Appl. Math. 26 (1973), 201-250.

8. Linear partial differential equations with constant coefficients, Gordon \& Breach, New York, 1956.

9. __ A link between local solvability of pseudodifferential operators and uniqueness in the Cauchy problem, Amer. J. Math. 94 (1972), 167-188.

10. __, Discrete phenomena in uniqueness in the Cauchy problem, Proc. Amer. Math. Soc. 46 (1974), 229-233.

11. M. Protter, The Cauchy problem for a hyperbolic second order equation, Canad. J. Math. 6 (1954), 542-553.

12. M. I. Viß̌k and V. V. Grußin, Elliptic pseudodifferential operators on a closed manifold which degenerate on a submanifold, Soviet Math. Dokl. 10 (1969), 1316-1320.

Department of Mathematics, University of Alabama, Birmingham, AlaBAMA 35294 